International Journal of Engineering, Science and Technology

Vol. 9, No. 2, 2017, pp. 26-38 \begin{tabular}{c}
\hline \hline INTERNATIONAL \\
JOURNAL OF \\
ENGINEERING, \\
SCIENCE AND \\
TECHNOLOGY \\
\hline \hline
\end{tabular}

www.ijest-ng.com

www.ajol.info/index.php/ijest

(C) 2017 MultiCraft Limited. All rights reserved

\title{
Modeling the wire-EDM process parameters for EN-8 carbon steel using artificial neural networks
}

\author{
Vaishali Sharma*, Manish Kumar Sagar \\ Department of Mechanical Engineering, Madhav Institute of Technology \& Science, Gwalior, INDIA \\ *Corresponding Author: e-mail: sharmavaishali476@gmail.com (Vaishali Sharma), manishsagar@gmail.com (Manish Kumar Sagar)
}

\begin{abstract}
Accompanying the evolution of mechanical industry, the need for alloy materials and ceramics as well, having high toughness, hardness \& impact resistance are also increasing. Wire-EDM process is a quick fix to this box. As this WEDM process is proficient in machining these materials meticulously, regardless to their toughness \& hardness. The present work discusses the experimental study on wire-cut machining of EN8 Carbon Steel. This paper presents an Artificial Neural Network approach in a multi objective optimization problem, for predicting the performance measure of a machining process. The input neurons were designed by Taguchi Orthogonal Array with 5 levels each with the help of software Minitab 17. In this research work, a comparative study for predicting multiple response parameters have been carried out using Feed forward and Cascade forward back propagation algorithms of ANN. This study was conducted by varying the network designing in each case. Two different back-propagation training algorithms were used in neural network designing such as Powell-Beale Conjugate Gradient (traincgb) and Levenberg-Marquardt (trainlm). Predictive accuracy of both the models was evaluated using Mean Square Error (MSE), Root Mean Square Error (RMSE) and coefficient determination $\left(\mathrm{R}^{2}\right)$. The outcomes proved that both the algorithms were able to produce feasible models. Cascade model outperformed with all statistical tests between both.
\end{abstract}

Keywords: Artificial Neural Network, Feed forward back propagation, Cascade forward back propagation

DOI: http://dx.doi.org/10.4314/ijest.v9i2.3

\section{Introduction}

For decades, non-traditional machining (NTM) techniques, such as electrochemical machining, abrasive jet machining, electrical discharge machining and, to some extent, laser and ultrasonic machining has been considered as ancillary processes and generally been hired when traditional ways failed to deliver. Remarkably enhanced mechanical, thermal and chemical properties of modern materials gave rise to the need of unconventional methods as those were unable to machine by traditional ways. Wire cut Electrical Discharge Machining (WEDM) is such an unconventional machining process, used extensively to produce a complex intrinsic and extrinsic irregular shaped job very precisely with a difficult to machine, electrically conductive material like ceramics, composites etc. CNC wire cut EDM establishes an impulse voltage between cathode and anode of impulse source, which is restrained by a servo system, to get a certain gap so as to realize impulse discharging between cathode and anode. Workpiece behaves as anode and wire electrode as cathode of impulse power source and both remain immersed in a dielectric medium. When wire electrode is approaching the workpiece and the gap between them attains a certain value, the dielectric medium breakdown occurs and electrical discharging takes place. Plenty of tiny holes results due to erosion of impulse discharging and thus delivers the desired shape of workpiece. The eroded workpiece cools down and flushed away by the dielectric fluid.

WEDM process is guided by numerous process parameters such as pulse off time, pulse on time, discharge current, pulse duration, polarity, servo reference voltage, feed rate, flushing pressure, operating current, etc. Each process parameter dominates the machining accuracy in some way and lot of research has been conducted regarding the optimization and modeling of these process parameters. Mathew et al. (2014) predicted the performance characteristics namely Material removal rate (MRR) and Surface roughness (SR) by using ANN models that were developed by using back propagation algorithms. Vasudevan et al. (2004) 
investigated the influence of machining parameters on the mechanism of MRR and SR and the trial runs were performed based on Taguchi's single objective optimization technique. Roberts (2017) established the mathematical model of wire lateral vibration in machining process in order to study the kerf variations in micro-WEDM and obtained its analytical solution. Badde et al. (2012) interrogated the effects of process variables on machining performance by an Adaptive neuro-fuzzy inference system (ANFIS). Di et al. (2009) practiced an integrated approach, principal component analysis (PCA) coupled with Taguchi's robust theory for simultaneous optimization of multiple responses of WEDM process for machining a metal matrix composites (MMC). The chosen performance indicators were SR, MRR, wire wear ratio, white layer thickness and kerf width. Goyal and Goyal (2011) varied pulse off time, pulse on time, servo voltage, wire feed, wire tension and dielectric pressure during tests on AISI304 to investigate their effect on MRR, SR, and dimensional deviation by Taguchi Grey relational analysis.

In this paper, ANN technique is used to predict the response measures at various input combinations. The main objective of this paper is to compare and explore the feasibility of feed forward and cascade forward back propagation algorithms in ANN models for predicting the process responses such as kerf width, over cut, dimensional deviation and MRR. In non-traditional machine tools, generally trial and error method is employed to optimize the process parameters so as to optimize the machining time and material wastage. This present work offers a significant way to reduce the wastes from the workspace. By using artificial networks, a machine can be trained appropriately with a specified set of experimental data which will in turn gives the very accurate results for any unseen data without even performing the actual experiment, which will eventually reduce the experimental cost and time of production, which plays a crucial role in success of any manufacturing industry. The accuracy of results relies on how well the training has been done. The neural networks has been developed with the help of MATLAB 8.1 (R13) package and the data required for training and testing the ANN are obtained by conducting the trial runs on a wire cut EDM machine.

\section{Experimental Details}

\subsection{Materials and Method}

The experiments were conducted on a computer numerically controlled CHMER-EDM (CW-53GF) equipped with W5F system software with accuracy of $5 \mu$, manufactured by Ching Hung Machinery \& Electric Industrial Co. Ltd. Distilled water is taken as dielectric medium having dielectric conductivity of $1.0 \mathrm{~K} . \mathrm{cm}$. The machine unit is shown in Figure 1. In this WEDM process, a spark is established in the close proximity between the continuously travelling brass wire $(0.25 \mathrm{~mm}$ diameter $)$ and the workpiece. The carbon steel EN8 of $40 \mathrm{~mm} \times 40 \mathrm{~mm} \times 40 \mathrm{~mm}$ size has been used as a workpiece material in present investigation. EN8 has been selected as a workpiece by taking into account its various engineering applications such as in shafts, bolts, studs, connecting rods etc. The workpiece was machined until the depth of $5 \mathrm{~mm}$. Chemical composition of EN8 is given in Table 1. The photograph of a machined workpiece is shown in Figure 2.

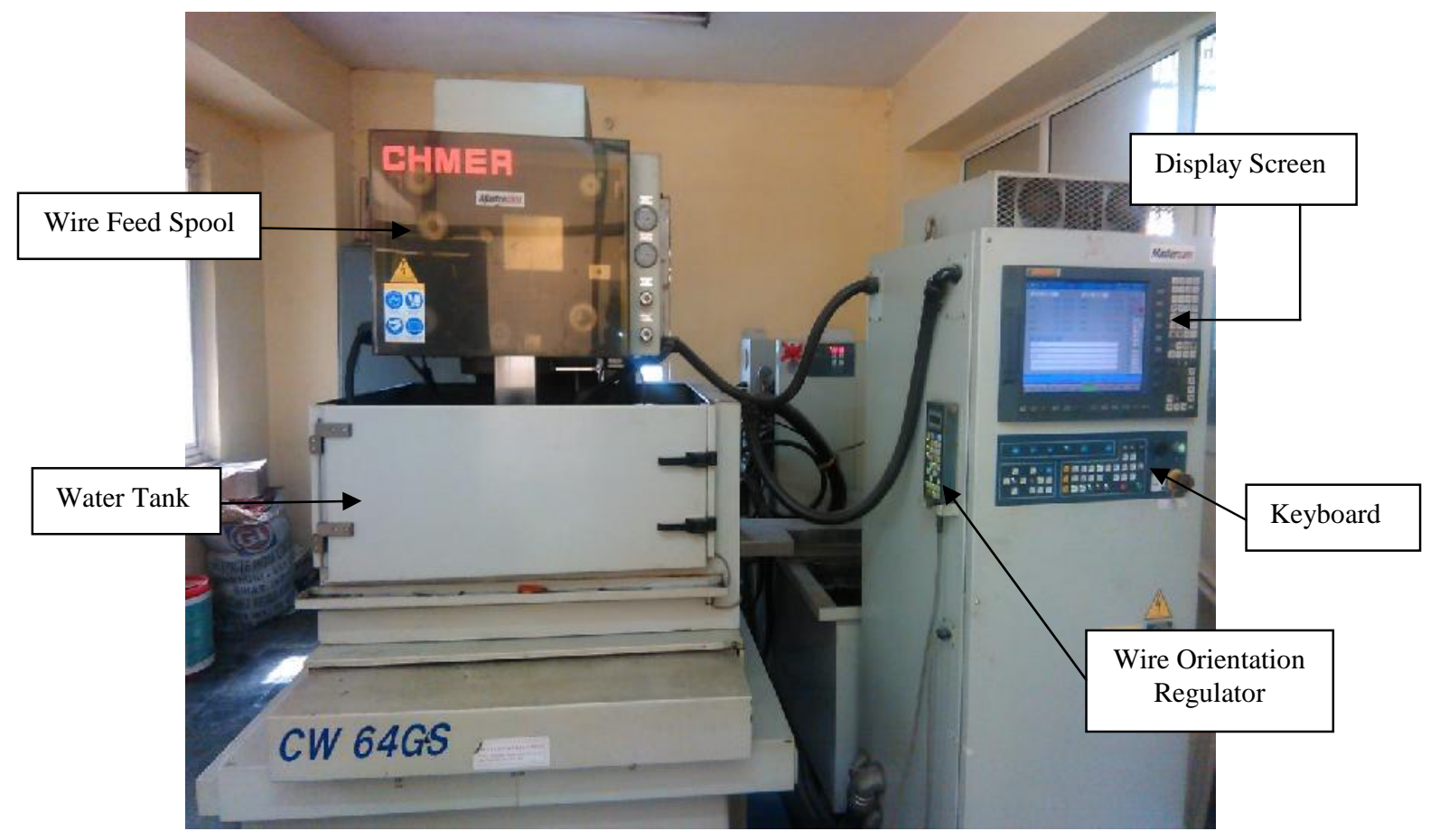

Figure 1. WEDM setup used for experimentation

Based on preliminary review Ton, Toff, WT, WF, SV were selected as the varying process parameters in this investigation and rest of the parameters were kept constant during the experiment. Cutting speed was digitally shown on the monitor of the machine 
and quantitatively in terms of $\mathrm{mm} / \mathrm{min}$. The kerf width was measured by stereozoom microscope. Overcut, dimensional deviation and MRR were calculated by the following expressions:-

Overcut $=($ width of cut $-\mathrm{D}) / 2$

Dimensional deviation $=(0.5 \times \mathrm{D})+$ overcut

MRR $=($ Cutting Speed $x \mathrm{~kW} x$ Height $)$

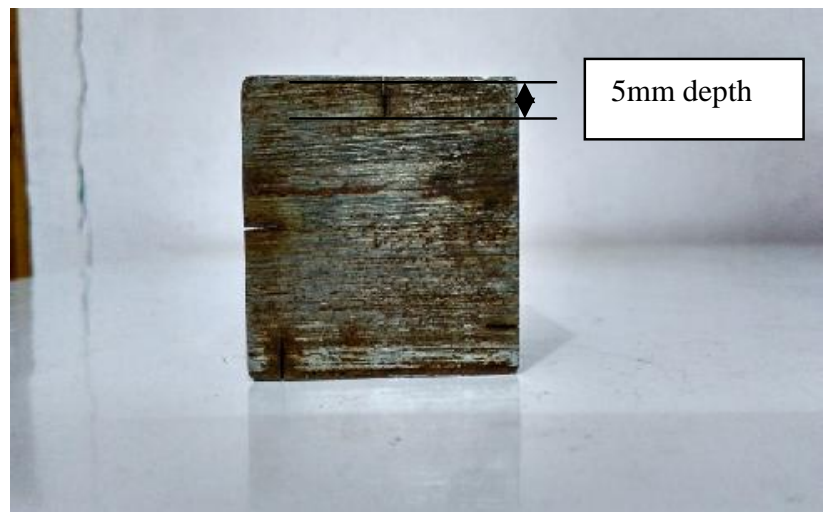

Figure 2. Final specimen showing5 $\mathrm{mm}$ deep grooves on all 4 faces

Table 1. Chemical composition of EN8

\begin{tabular}{cccccccc}
\hline Element & $\begin{array}{c}\text { Carbon } \\
(\mathbf{C})\end{array}$ & $\begin{array}{c}\text { Silicon } \\
(\mathbf{S i})\end{array}$ & $\begin{array}{c}\text { Manganese } \\
(\mathbf{M n})\end{array}$ & $\begin{array}{c}\text { Phosphorus } \\
(\mathbf{P})\end{array}$ & $\begin{array}{c}\text { Sulphur } \\
(\mathbf{S})\end{array}$ & $\begin{array}{c}\text { Chromium } \\
(\mathbf{C r})\end{array}$ & Nickel (Ni) \\
\hline Content $(\%)$ & 0.032 & 0.213 & 0.495 & 0.030 & 0.030 & 0.094 & 0.044 \\
\hline
\end{tabular}

\subsection{Design of experiments}

Classical experimental design methods are sometimes found a bit too complex and difficult to use when there are large number of variables involved. As the number of variable increases the number of experiment that has to be carried out also increases. To conduct the experiments within the given time and cost constraint, Taguchi method was used in this study to plan the experiments wisely. Five levels have been decided for each of the five process parameters in the present experimental work. Minitab 17 was used for Taguchi analysis. The process parameters and their selected levels are listed in Table 2. Taguchi method based $L_{25}$ orthogonal array has been used for experimental design (Table 3).

Table 2. Machining settings used in the experiments

\begin{tabular}{llccccc}
\hline $\begin{array}{l}\text { Machining } \\
\text { parameter }\end{array}$ & Symbol & Level 1 & Level 2 & Level 3 & Level 4 & Level 5 \\
\hline Pulse-on Time $(\mu \mathrm{s})$ & A & 3 & 4 & 5 & 6 & 7 \\
Pulse-off Time $(\mu \mathrm{s})$ & B & 10 & 15 & 20 & 24 & 30 \\
Wire Tension $(\mathrm{N})$ & $\mathrm{C}$ & 4 & 7 & 9 & 11 & 13 \\
Wire Feed $(\mathrm{mm} / \mathrm{s})$ & $\mathrm{D}$ & 3 & 6 & 10 & 12 & 13 \\
Servo Voltage $(\mathrm{V})$ & $\mathrm{E}$ & 15 & 20 & 25 & 30 & 35 \\
\hline
\end{tabular}

Table 3. Experimental layout using L25 orthogonal array and experimental results

\begin{tabular}{cccccccccc}
\hline $\begin{array}{c}\text { Experiment } \\
\text { no. }\end{array}$ & Ton & Toff & WT & WF & SV & $\begin{array}{c}\text { KW } \\
(\mathrm{mm})\end{array}$ & $\begin{array}{c}\text { OC } \\
(\mathrm{mm})\end{array}$ & $\begin{array}{c}\text { DD } \\
(\mathrm{mm})\end{array}$ & $\begin{array}{c}\text { MRR } \\
\left(\mathrm{mm}^{3} / \mathrm{m}\right)\end{array}$ \\
\hline 1. & 1 & 1 & 1 & 1 & 1 & 0.297 & 0.0235 & 0.1485 & 9.6085 \\
2. & 1 & 2 & 2 & 2 & 2 & 0.275 & 0.0125 & 0.1375 & 2.9458 \\
3. & 1 & 3 & 3 & 3 & 3 & 0.297 & 0.0235 & 0.1485 & 3.5640 \\
4. & 1 & 4 & 4 & 4 & 4 & 0.300 & 0.0250 & 0.1500 & 3.4080 \\
5. & 1 & 5 & 5 & 5 & 5 & 0.295 & 0.0225 & 0.1475 & 3.0916 \\
6. & 2 & 1 & 2 & 3 & 4 & 0.271 & 0.0105 & 0.1355 & 4.3360 \\
7. & 2 & 2 & 3 & 4 & 5 & 0.298 & 0.0240 & 0.1490 & 4.9563 \\
8. & 2 & 3 & 4 & 5 & 1 & 0.295 & 0.0225 & 0.1475 & 4.6256 \\
9. & 2 & 4 & 5 & 1 & 2 & 0.297 & 0.0235 & 0.1485 & 5.2980 \\
10. & 2 & 5 & 1 & 2 & 3 & 0.287 & 0.0185 & 0.1435 & 3.7080 \\
11. & 3 & 1 & 3 & 5 & 2 & 0.300 & 0.0250 & 0.1500 & 6.0000
\end{tabular}


Table 3 (cont'd). Experimental layout using L25 orthogonal array and experimental results

\begin{tabular}{cccccccccc}
\hline $\begin{array}{c}\text { Experiment } \\
\text { no. }\end{array}$ & Ton & Toff & WT & WF & SV & $\begin{array}{c}\text { KW } \\
(\mathrm{mm})\end{array}$ & $\begin{array}{c}\text { OC } \\
(\mathrm{mm})\end{array}$ & $\begin{array}{c}\text { DD } \\
(\mathrm{mm})\end{array}$ & $\begin{array}{c}\text { MRR } \\
\left(\mathrm{mm}^{3} / \mathrm{m}\right)\end{array}$ \\
\hline 12. & 3 & 2 & 4 & 1 & 3 & 0.275 & 0.0125 & 0.1375 & 5.4098 \\
13. & 3 & 3 & 5 & 2 & 4 & 0.280 & 0.0150 & 0.1400 & 5.6780 \\
14. & 3 & 4 & 1 & 3 & 5 & 0.372 & 0.0610 & 0.1860 & 5.2970 \\
15. & 3 & 5 & 2 & 4 & 1 & 0.368 & 0.0590 & 0.1840 & 6.3590 \\
16. & 4 & 1 & 4 & 2 & 5 & 0.295 & 0.0225 & 0.1475 & 5.9000 \\
17. & 4 & 2 & 5 & 3 & 1 & 0.275 & 0.0125 & 0.1375 & 5.4450 \\
18. & 4 & 3 & 1 & 4 & 2 & 0.324 & 0.0370 & 0.1620 & 5.1840 \\
19. & 4 & 4 & 2 & 5 & 3 & 0.324 & 0.0370 & 0.1620 & 8.8250 \\
20. & 4 & 5 & 3 & 1 & 4 & 0.350 & 0.0500 & 0.1750 & 8.6120 \\
21. & 5 & 1 & 5 & 4 & 3 & 0.287 & 0.0185 & 0.1435 & 4.9620 \\
22. & 5 & 2 & 1 & 5 & 4 & 0.311 & 0.0305 & 0.1555 & 4.9760 \\
23. & 5 & 3 & 2 & 1 & 5 & 0.367 & 0.0585 & 0.1835 & 7.3400 \\
24. & 5 & 4 & 3 & 2 & 1 & 0.300 & 0.0250 & 0.1500 & 5.1336 \\
25. & 5 & 5 & 4 & 3 & 2 & 0.361 & 0.0555 & 0.1805 & 5.7760 \\
\hline
\end{tabular}

\section{Research Methodology}

Function estimation is the task of learning and constructing a function that produces the same yield from the input vectors based on the available training data (Maher et al., 2015). Artificial Neural Network (ANN) approach is a captivating mathematical tool, which can be used to replicate a wide variety of engineering and complex scientific problems into simpler ones. In the last few years, this ANN approach had become an eventual tool for various engineering applications, due to its greatly reliable prediction ability. In order for the network to replicate a desired behavior, the parameters of the network should be optimized through the appropriate learning process. An ANN is a guided graphical arrangement, where nodes execute some computations which may be either very simpler ones (such as summing up the inputs) or sometimes quite complicated (a node may contain another neural network). Each connection determines the information (signal) flow from one node to another usually associated by a number called as weight, which represents the degree to which an information (signal) is being weaken or amplified by a connection. But only those graphs are termed as neural network whose weights are primarily random and the learning algorithm suggests the value of weights that will attain the preferred task.

The type of neural network architecture depends on the ways nodes are linked to each other. There are quite a few node functions such as sigmoid, ramp, step, Piecewise linear function, etc and learning algorithms such as hebbian learning, back propagation learning, competitive learning etc. and architecture such as fully connected network, feed-forward network, acrylic networks, modular neural networks, etc., are available (Rao and Krishna, 2013; Maher et al., 2015). In present study, back-propagation neural network (BPNN) has been adopted due to its capability of giving fast response and high learning accuracy. It provides a computationally efficient method for training the network. An optimum network architecture and pertinent number of training cycles i.e. epochs at different input combinations will need to be established in order to improve the network performance. So, in this study we compared two types of ANN network for prediction of various response parameters. First network is Feed forward back propagation and second is Cascade forward back propagation. So far very petty literatures are available on comparison of networks in ANN. Hence, this work is an attempt to do the comparative study of the networks and evaluate the performance of each network for selecting the best one out.

\section{ANN Model Development and Training}

This part will demonstrate how to create an Artificial Neural Network (ANN) by using the Neural Network Toolbox in MATLAB. A few steps will need to be followed to make such a neural network that is useful to predict the responses:

(a) Database Collection: establishing the data by conducting experiments on EN8 at different machining conditions.

(b) Develop the input and output for the data.

(c) Transfer the above data from Excel file to MATLAB file.

(d) Establish the network in terms of choosing the architecture of ANN, training algorithms, training functions and parameters of the network.

(e) Train the network with the given data.

(f) Test the trained ANN to evaluate the network performance.

(g) Now, Simulation and Prediction will be performed using the trained network.

\subsection{Data sampling}


The dataset comprises of experimentally obtained 25 observations. The dataset was then randomly divided into subsets, namely training set which contains 20 observations i.e. $80 \%$ of the total observations and secondly the test set containing the rest 5 observations i.e. $20 \%$ of the total observations.

\subsection{Artificial neural network models}

4.2.1 Feed forward back propagation (FB) model

In Feed forward model shown in Figure. 3, the neurons are arranged in layers, with the first layer receiving the input and the last layer producing the output. These networks possess the output layer of linear neurons, more often preceded by one or more hidden layers of sigmoid neurons. The linear output layer lets the network to produce the values outside the range -1 to +1 . The informations in these networks are constantly fed forward from one layer to another and this justifies their name as feed forward network. Learning in these feed-forward networks belongs to the realm of supervised learning, in which the pairs of input and output values are fed into the network for many cycles, so as the network 'learns' the relationship between the input and output (Mehrotra et al., 1997). Back propagation learning algorithm is used for learning these networks. During training, calculations are carried out from input layer directed to output layer, and the errors are then propagated from the output layer to the preceding layers. These networks are well proficient to learn linear and non-linear relationships between inputs and outputs.

In order to achieve leading results, different training algorithms have been attempted in both the models. In this feed forward model, Powell-Beale Conjugate Gradient (traincgb) and Gradient descent with momentum weight and bias learning function (learngdm) has been employed as training and learning function respectively. Networks with single hidden layers topologies were used in both the models.

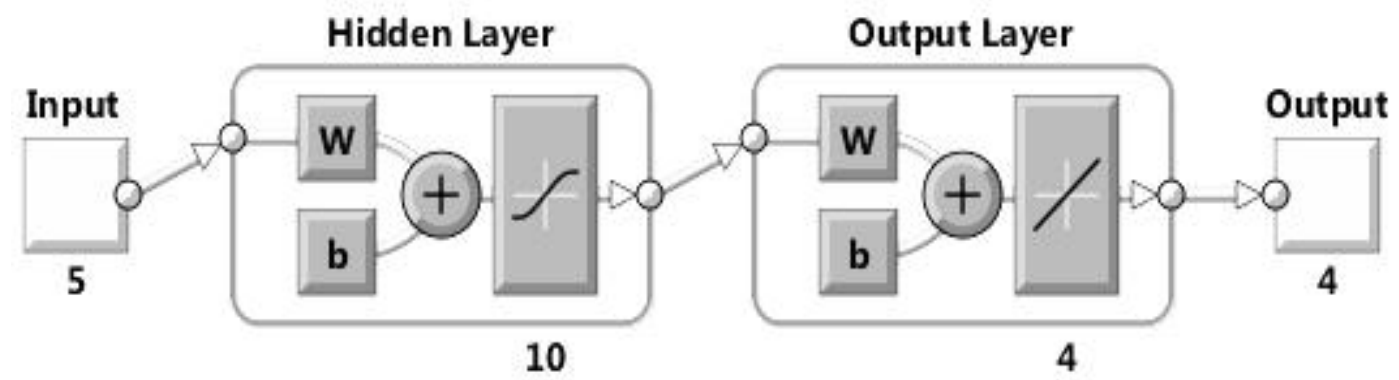

Figure 1:- Feed Forward Back Propagation Network.

\subsubsection{Cascade forward (CF) model}

Cascade forward back propagation model shown in Figure. 4 is similar to feed forward models but it includes a weight connection from input to each layer and from every previous layer to the following layers. The function 'newcf "creates a cascade forward network in MATLAB. For example, a four layer network will have connections from input to all the four layers. This network will also have connections from layer 1 to layer 2, layer 2 to layer 3 , layer 3 to layer 4 and layer 1 to layer 4 . These connections improve the speed at which the network learns the desired relationship. CF models are similar to feed forward back propagation network in using the back-propagation algorithm for weights updating.

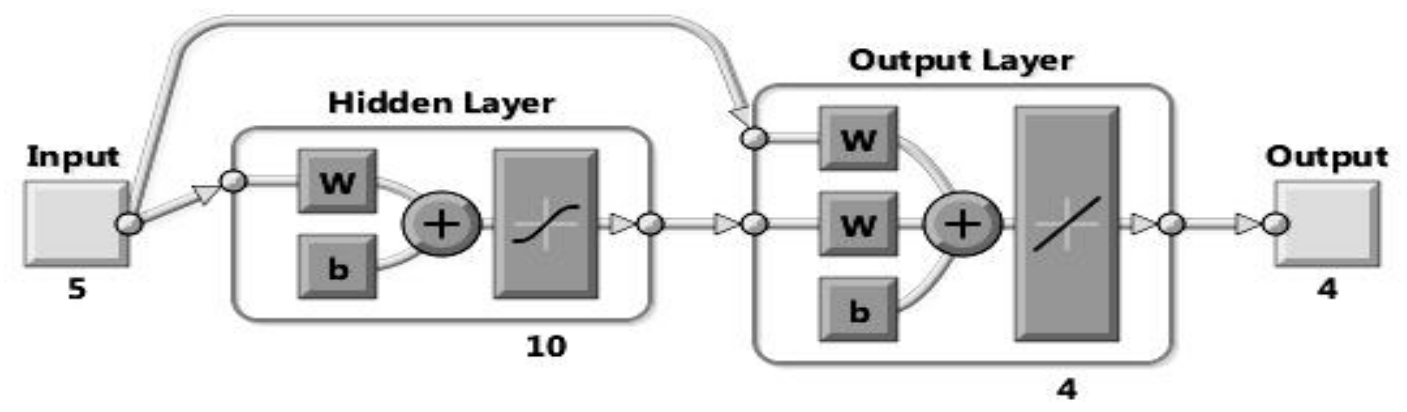

Figure 2. Cascade Forward Back Propagation Network.

Levenberg-Marquardt (trainlm), training function has been adopted in this network. Rest all the functions are same. For both the networks, linear transfer function (purelin) and tan-sigmoid transfer function (tansig) were used for output layer and hidden layers respectively. Linear transfer function has been adopted in output layer so that the values of the output are not limited between 0 and +1 as by using tan-sigmoid transfer function in hidden layers. The performance of both the networks was evaluated by using MSE, RMSE \& $\mathrm{R}^{2}$. 


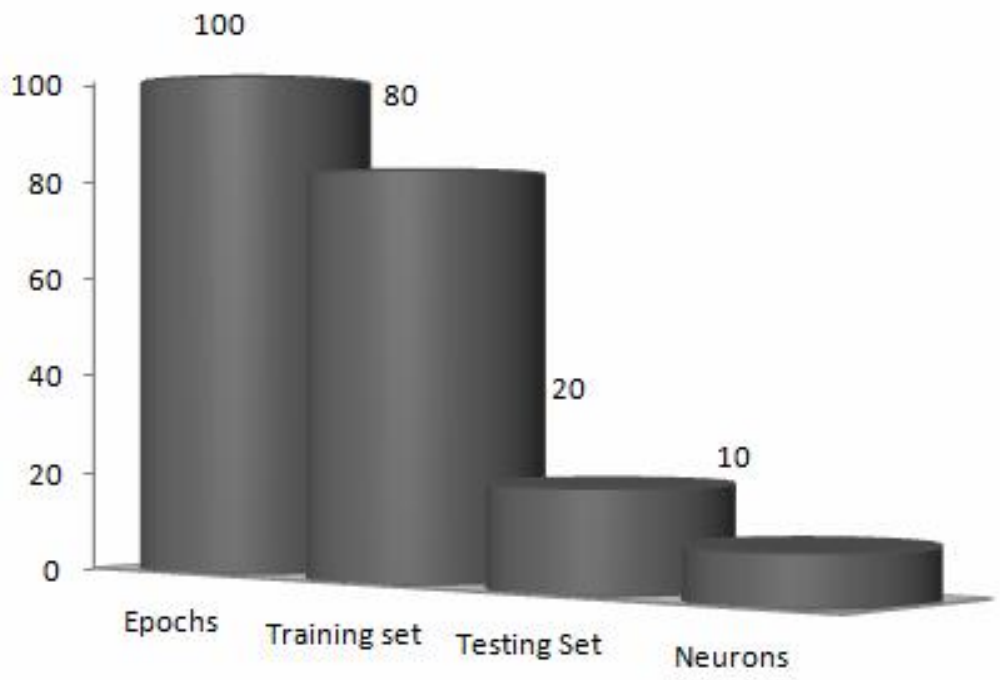

Figure 5. Parameters used in Artificial Intelligence Model

The datasets were divided into two disjoint subsets $80 \%$ for training and $20 \%$ for testing. The number of neurons in hidden layer was selected upto 10 as shown in Figure 5. Biases and weights were randomly initialized. Weights are the information used by a neural network to solve a problem. The networks were trained upto 100 epochs.. In this research, two neural networks have been developed by employing feed forward and cascade forward back propagation techniques.

\section{Training pattern of ANN's}

Based on the way the networks get trained, the artificial neural networks are categorized into two categories: supervised and unsupervised. In supervised learning, the networks are trained by providing both input and output data patterns. In unsupervised learning, there are no previously known answers, these networks are provided only with inputs. Such networks must develop their input stimuli representation on its own. These given inputs are the training data samples. The neural networks are able to calculate and adjust the connection weights via different ways so as to reduce the errors in the network.

The basic framework for training a neural network is shown in Figure. 6 (Ramakrishnana and Karunamoorthy, 2008).

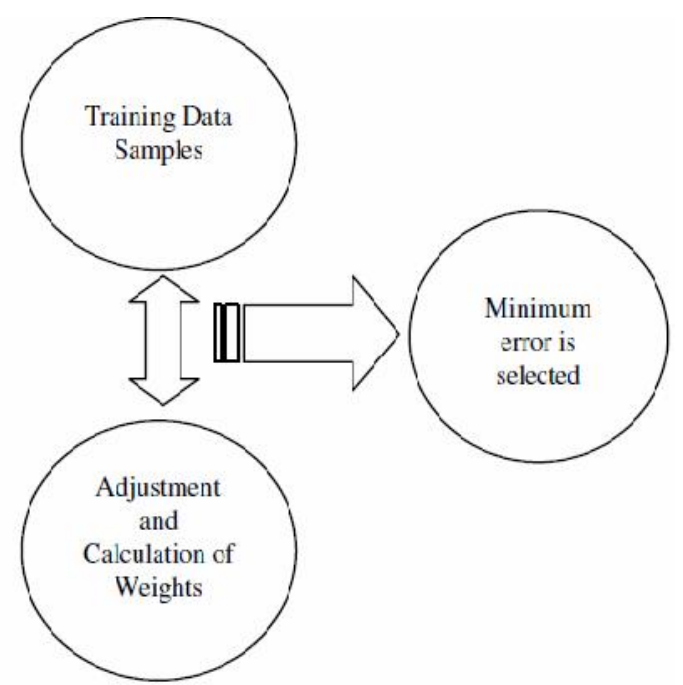

Figure 6. Training pattern of ANN

\section{Results \& Discussion}

After the networks are trained, tested and evaluated successfully, the best network needs to be determined which gives the best result and minimized error. So after analyzing, the developed cascade back propagation models resulted in better predictions of machining responses and its results then can be compared with the experimental results and along with the feed forward back propagation models. Table 4 depicts the predicted results during testing for all the responses by both cascade forward and feed forward back propagation algorithms. The predicted values from both the algorithms are quite closer but the cascade forward 
model gave the results much closer to the experimental values. On the other hand, ANN shows the lack of consistency in feed forward algorithm. This could be due to non-linear experimental data that lead to this problem.

The predicted response values for all the machining responses with respect to the stated process parameters are shown in figure $6,7,8, \& 9$. The results indicated that the ANN's ability in predicting the responses is excellent in Cascade back propagation algorithm than in Feed forward. Figures 6 to 9 show the comparison of measured and predicted responses from both the algorithms. The cascade predicted results were found to be very closer to the experimental values. During training the following training windows shown in Figures 10, 11(a) and (b), 12, 13, 14, 15, \& 16 opens. These windows display the training progress and allow you to halt the training at any point by clicking stop training. A figurative comparison of predicted results to that of measured results by both Feed and Cascade forward back propagation algorithms for all the four response parameters during training are shown in Figures 6, 7, 8 \& 9.

Table 1. Predicted results of machining performances by feed forward and cascade forward back propagation algorithm

\begin{tabular}{|c|c|c|c|c|c|c|c|}
\hline \multicolumn{3}{|c|}{ Feed Forward Back Propagation } & \multicolumn{3}{c|}{ Cascade Forward Back Propagation } \\
\hline KW & OC & DD & MRR & KW & OC & DD & MRR \\
\hline 0.3105 & 0.0274 & 0.1448 & 5.6064 & 0.2793 & 0.0234 & 0.1478 & 4.7425 \\
\hline 0.3297 & 0.0254 & 0.1367 & 5.4537 & 0.3234 & 0.0335 & 0.1662 & 4.8498 \\
\hline 0.3074 & 0.0486 & 0.1429 & 5.843 & 0.3245 & 0.0575 & 0.1554 & 7.2839 \\
\hline 0.3094 & 0.0277 & 0.1435 & 4.2988 & 0.3309 & 0.0203 & 0.1609 & 5.2144 \\
\hline 0.3264 & 0.0457 & 0.1685 & 5.987 & 0.3284 & 0.0629 & 0.1775 & 5.7718 \\
\hline
\end{tabular}

Training state, Regression plot and Performance plot for both the models are shown in successive Figures 10, 11, 12, 13, 14 \& 15. Validation is employed to evaluate the network generalization $\&$ to hinder the training when generalization ceases to improve. Fig $10 \& 11$ depicts the training state of both FB and CF models. Validation tends to stop when GRADIENT performance decreases and the validation performance (VAL FAIL) increases. The minimum gradient emerged out as 0.00075873 at epoch 100 and $1.3742 \mathrm{e}-11$ at epoch 15 for $\mathrm{FB}$ and $\mathrm{CF}$ models respectively. At this point when validation ceases, the best validation performance score were recorded for both models as 0.026028 at time 1 and 0.0056799 at time 0 . as depicted in Figures $12 \& 13$. The MSE is the mean of square of differences between actual and target outputs. Lower MSE values allude better performance and zero signifies no error. The validation and test curves for both the cases are similar and in near proximity and the overall MSE recorded as $8.83003 \mathrm{e}^{-5}$ and $5.81779 \mathrm{e}^{-23}$. Figures $14 \& 15$ show the regression curves plotted between output and target values. Outputs predicted by the employed learning functions were plotted on y-axis against the given target values. The empty circle in the plot denotes the outputs and target relationships. In regression plots, one can witness, the outputs trace the targets very well for training,

Validation and testing for both the cases and the correlation measure $\mathrm{R}=0.99862$ and $\mathrm{R}=0.9962$ for $\mathrm{FB} \& \mathrm{CF}$ models respectively. Training continues till network's error starts reducing in respect of validation set. Different networks deviate from each other in terms of training functions employed. Thus, such networks can fix the multi dimensional riddle arbitrarily well, provided congruent data and enough neurons in its hidden layer as depicted in Figure 16. For conjugate gradient back propagation algorithm, the quest direction is repeatedly reset to the negative of the worked out gradient. This traincgb function can train any network only until the network inputs, its weights and transfer functions have derivative functions. Levenberg-Marquardt (trainlm) back propagation algorithm used for CF model, where training ceases automatically when generalization stops upgrading, as implied by an increase in mean square error (MSE) of the validation samples. Then the networks were simulated in the testing set.

\section{Prediction performance measures}

This part presents the numerical analysis of model prediction accuracies for the developed neural network models. The comparison between both the models was estimated by using mean square error (MSE), root mean square error (RMSE) \& coefficient of determination $\left(R^{2}\right)$. The lower the RSME, the more accurate the evaluation will be and the coefficient of determination $\left(R^{2}\right)$ measures the variance that is interpreted by the model, which is reduction of variance when using the model (Bobbili et al., 2015). These performance scales are the good measure of overall predictive accuracy. RMSE \& $\mathrm{R}^{2}$ yields the paradigm measures of predictive accuracy. All results recorded are for the training set and testing set. The predictive evaluation results are summarized in Table 5. The expressions of all the measures are given below.

$$
\mathrm{MSE}=\sum_{i=1}^{n}\left(\frac{Q_{\exp }-Q_{\text {pred }}}{n}\right)
$$



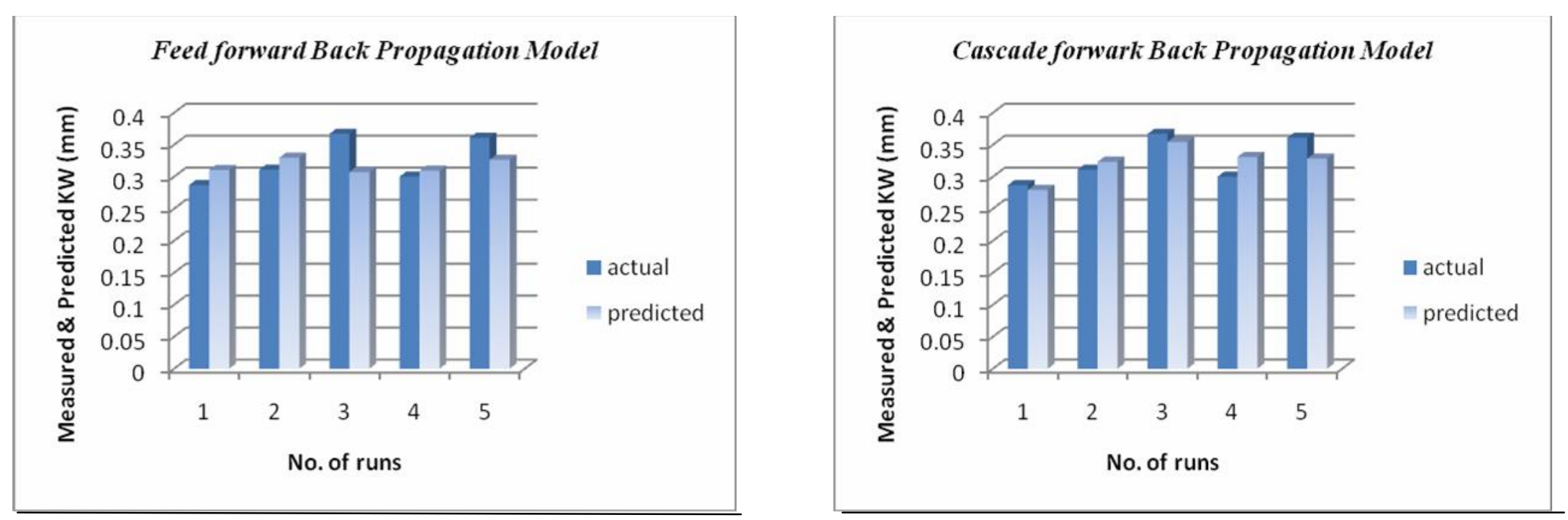

Figure 3. Comparison of predicted results of KW by Feed forward and Cascade forward back propagation algorithm.
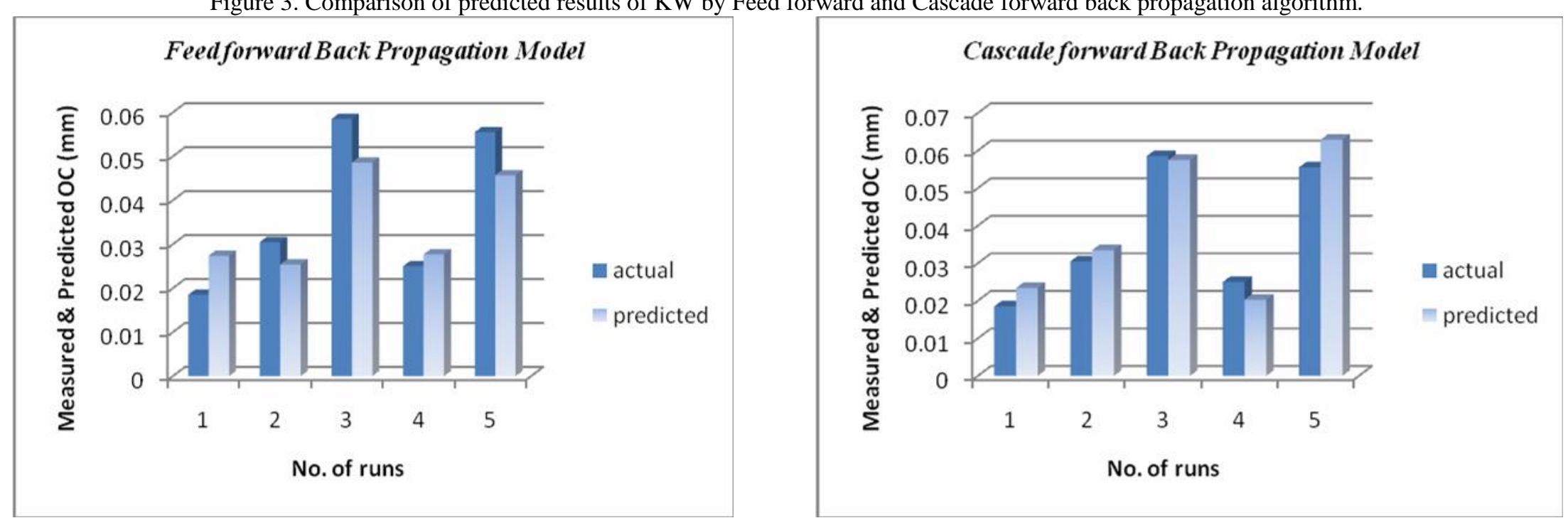

Figure 4. Comparison of predicted results of OC by Feed forward \& Cascade forward back propagation algorithm. 

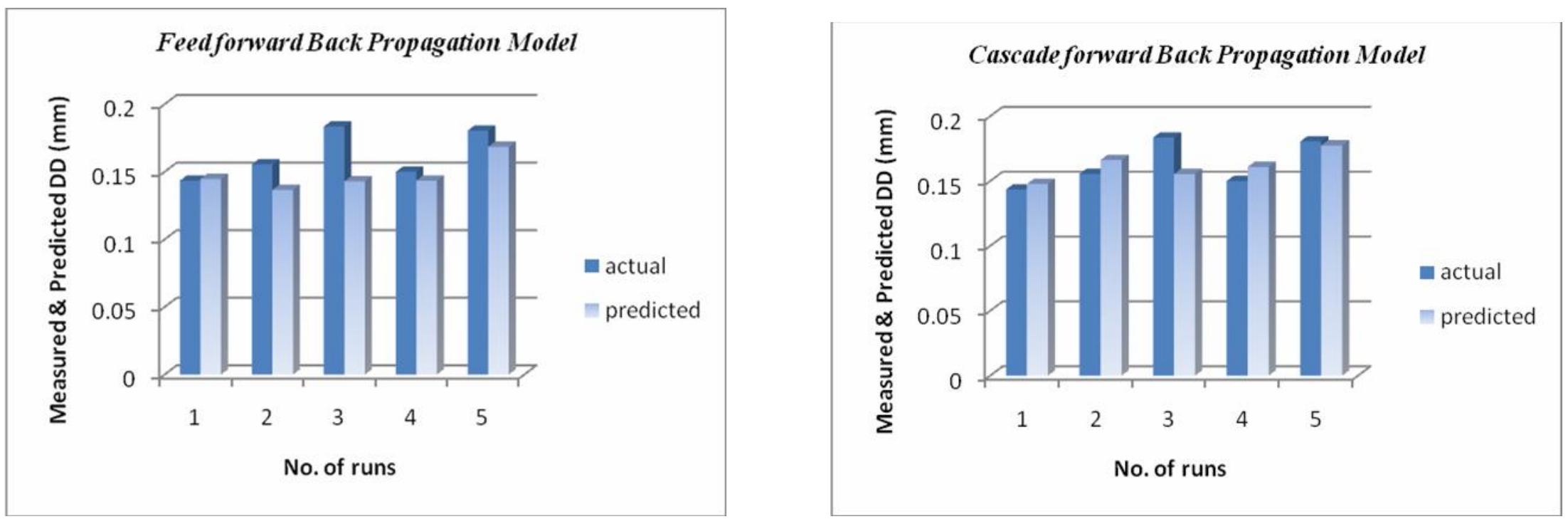

Figure 9. Comparison of predicted results of DD by Feed forward \&Cascade forward back propagation algorithm.
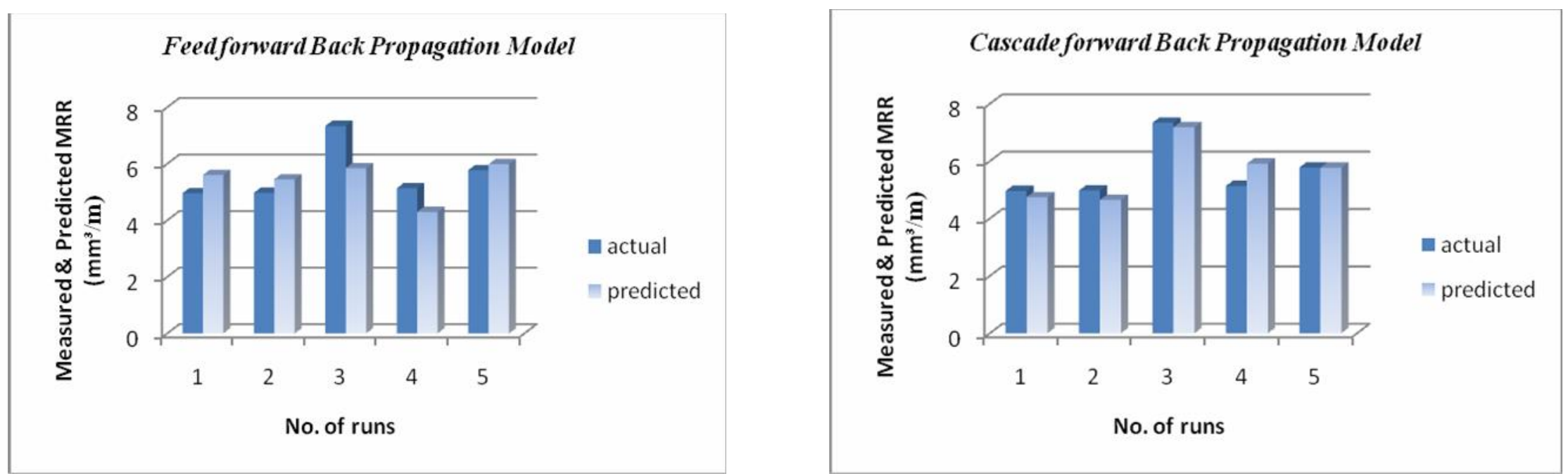

Figure 5. Comparison of predicted results of MRR by Feed forward \& Cascade forward back propagation algorithm. 


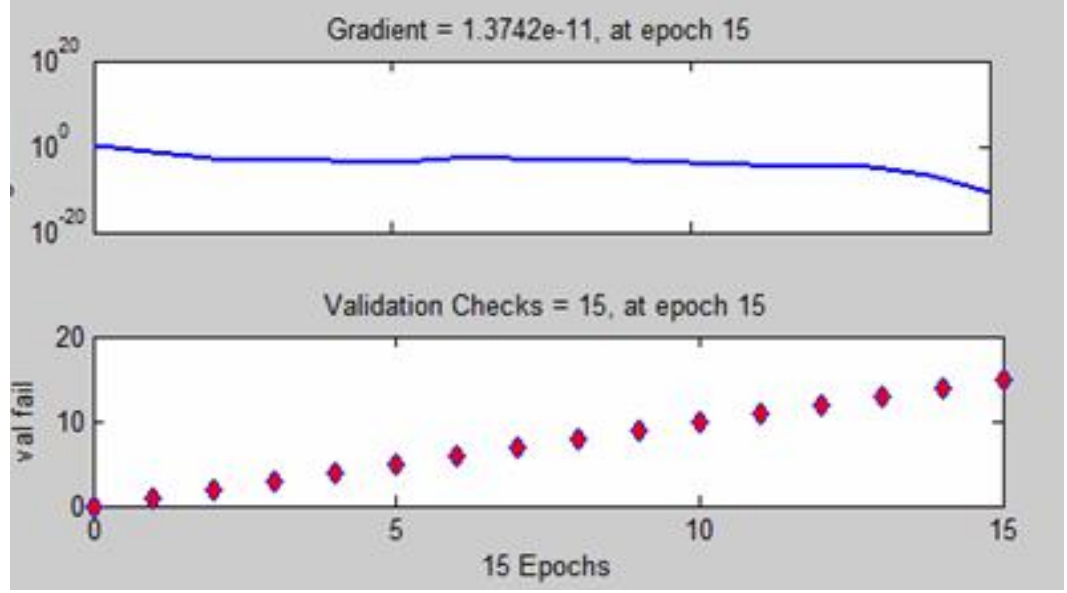

Figure 11(a). Training state of CF model.

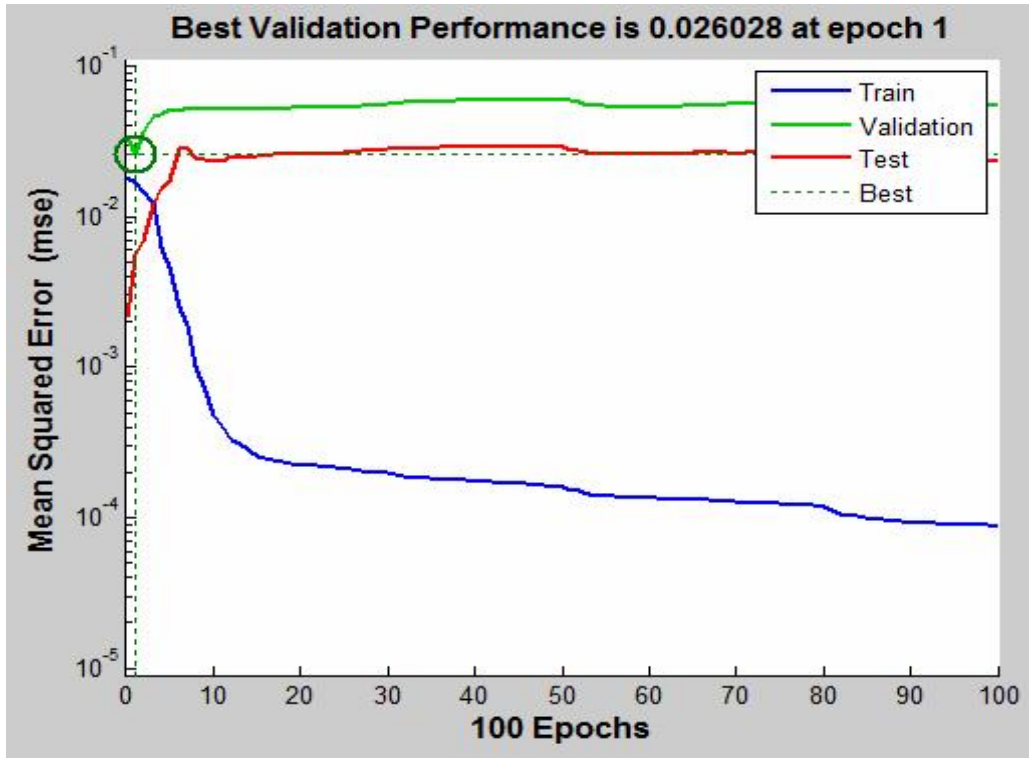

Figure 12. FB Network error values plot

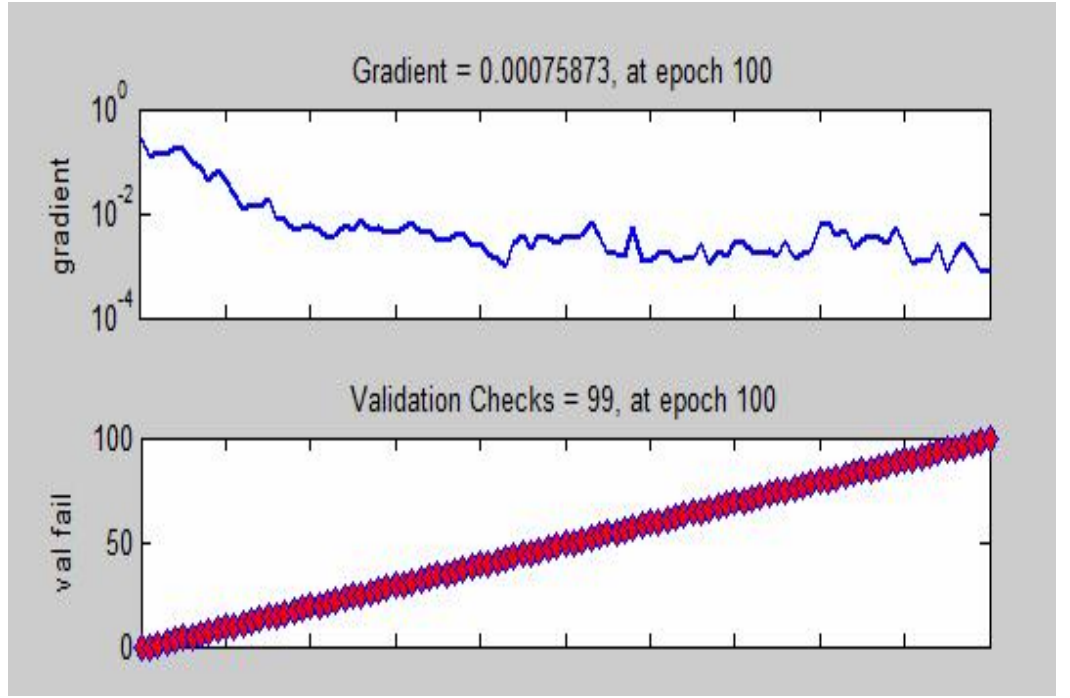

Figure 6(b) Training state of FB Model.

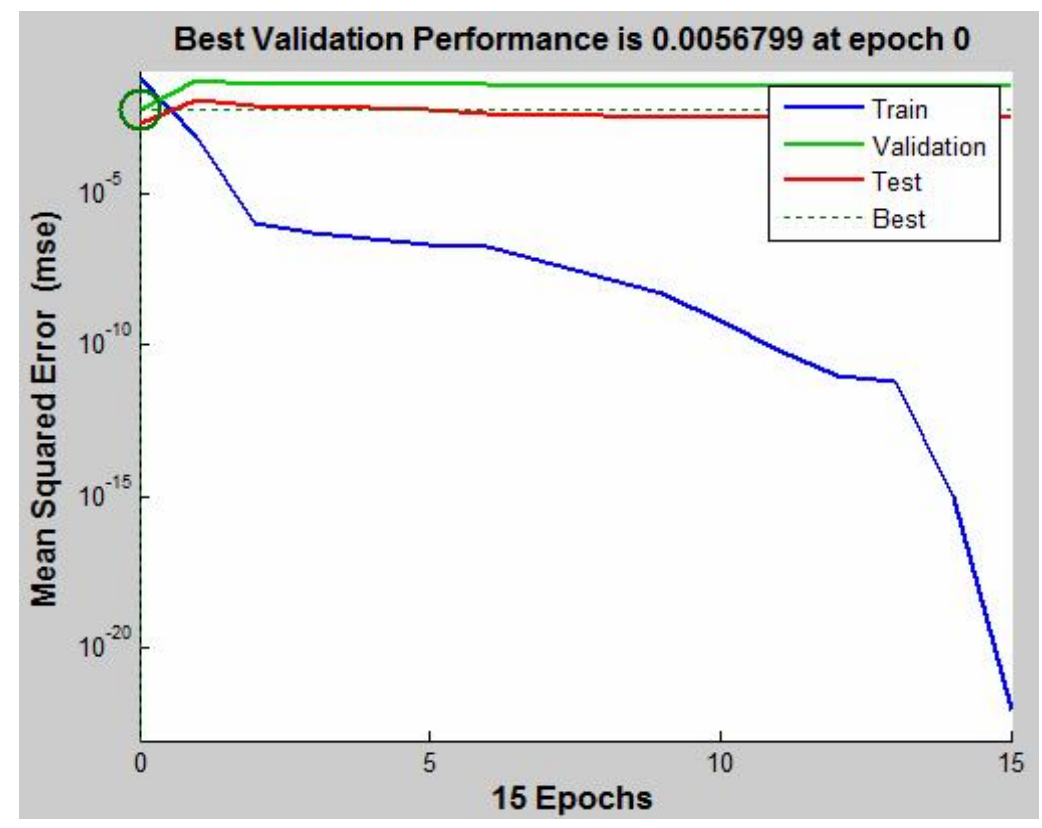

Figure 13. CF Network error values plot 

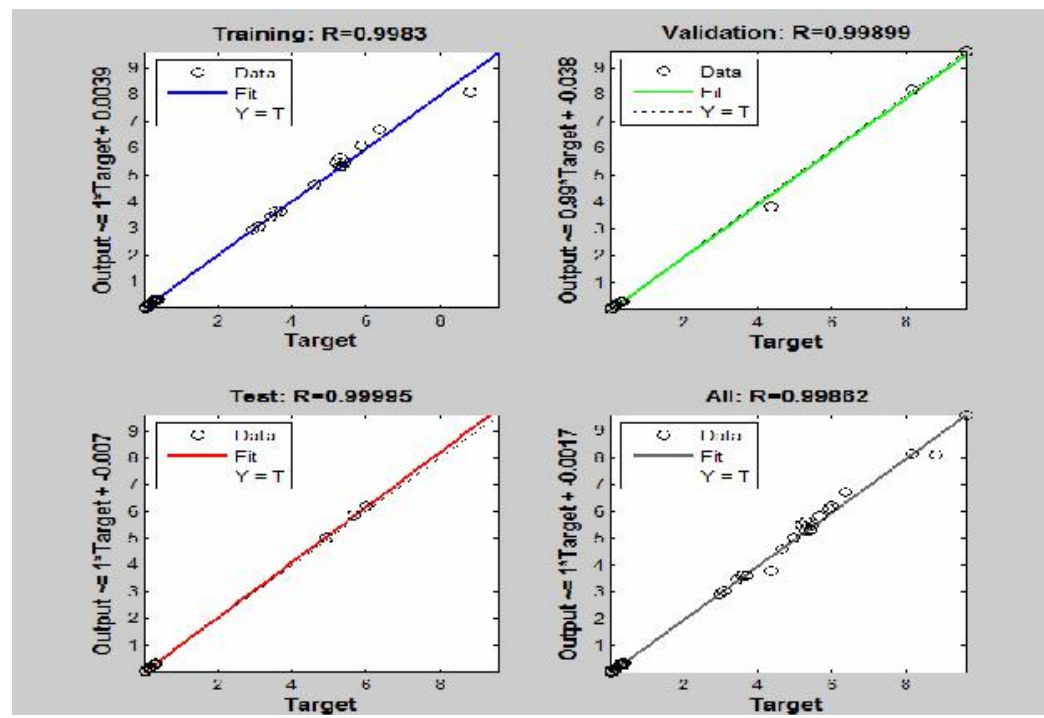

Figure 14. FB Network Regression Analysis
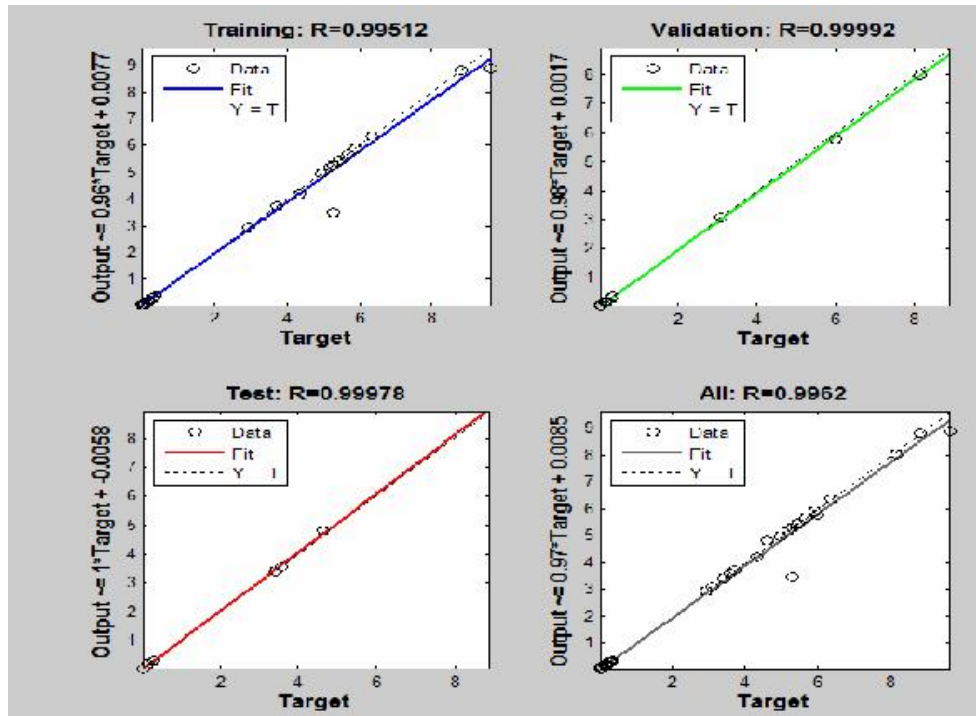

Figure 15:- CF Network Regression Analysis.

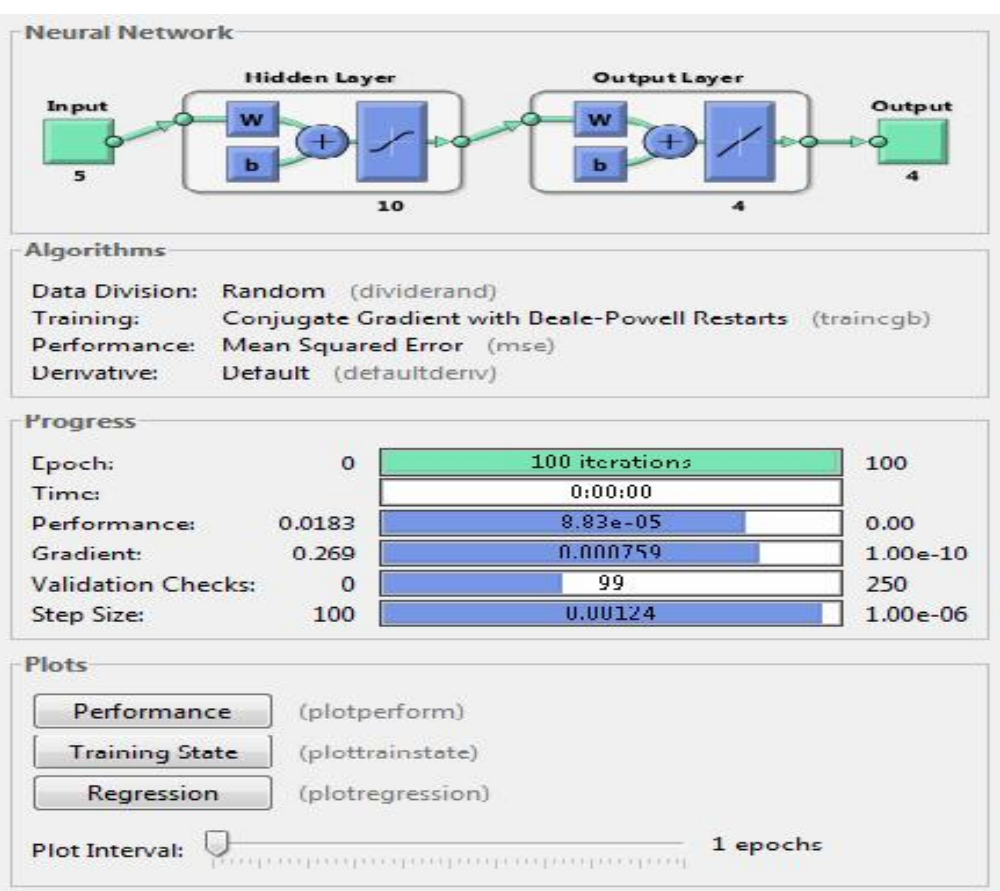

Opening Training State Plot

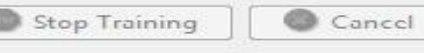

Figure 16:- Neural network training for feed forward network predictions 


$$
\begin{aligned}
& \text { RSME }=\sqrt{\frac{1}{n}\left[\sum_{i=1}^{n}\left(\frac{Q_{\text {exp }}-Q_{\text {pred }}}{Q_{\text {exp }}}\right)^{2}\right]} \\
& \mathrm{R}^{2}=1-\left[\sum_{i=1}^{n}\left(\frac{Q_{\text {exp }}-Q_{\text {pred }}}{Q_{\text {exp }}^{2}}\right)^{2}\right]
\end{aligned}
$$

where, $\mathrm{Q}_{\exp }=$ experimental value; $\mathrm{Q}_{\text {pred }}=$ predicted value; $\mathrm{n}=$ Number of observations in dataset.

Equations (1), (2) \& (3) were used to compare the prediction performance of developed models. The obtained MSE, RMSE \& $\mathrm{R}^{2}$ values of all the trained models are highly significant as well as the MSE, RMSE \& $\mathrm{R}^{2}$ values of tested CF model of DD are $4.2015 \times 10^{-6}, 0.0135 \& 0.9453$ are outstanding. The MSE, RMSE \& $\mathrm{R}^{2}$ values of tested CF models are comparatively better than FB models. These statistical tests signify the CF models more significant than the FB models which in turn demonstrate that the predictive accuracy of CF models outperforms the FB models. These statistical tests are summarized in Table 5.

\begin{tabular}{|c|c|c|c|c|c|c|c|}
\hline & \multicolumn{2}{|c|}{ MSE } & \multicolumn{2}{|c|}{ RMSE } & \multicolumn{2}{|c|}{$\mathbf{R}^{2}$} \\
\hline & & Train & Test & Train & Test & Train & Test \\
\hline \multirow[t]{2}{*}{ KW } & $\mathbf{C F}$ & $1.8413 \times 10^{-4}$ & $1.6147 \times 10^{-4}$ & 0.0384 & 0.0830 & 0.6845 & 0.5467 \\
\hline & FB & $2.3493 \times 10^{-3}$ & $2.2957 \times 10^{-4}$ & 0.0805 & 0.0967 & 0.8876 & 0.5555 \\
\hline \multirow[t]{2}{*}{ OC } & $\mathbf{C F}$ & $1.5275 \times 10^{-6}$ & $4.4344 \times 10^{-6}$ & 0.4035 & 0.1631 & 0.2078 & 0.5409 \\
\hline & FB & $2.1666 \times 10^{-4}$ & $1.2262 \times 10^{-5}$ & 0.4354 & 0.2571 & 0.5432 & 0.6433 \\
\hline \multirow[t]{2}{*}{ DD } & $\mathbf{C F}$ & $4.3161 \times 10^{-6}$ & $4.2015 \times 10^{-6}$ & 0.0492 & 0.0135 & 1 & 0.9453 \\
\hline & FB & $7.1962 \times 10^{-6}$ & $8.7588 \times 10^{-5}$ & 0.0737 & 0.1181 & 0.8796 & 0.8907 \\
\hline \multirow[b]{2}{*}{ M } & CF & $9.7977 \times 10^{-4}$ & $2.952 \times 10^{-3}$ & 0.0287 & 0.0198 & 0.7751 & 0.4512 \\
\hline & FB & $3.7205 \times 10^{-3}$ & 0.145 & 0.0517 & 0.1381 & 0.7980 & 0.8897 \\
\hline
\end{tabular}

Table 5.Accuracy of predictions for cascade \& feed forward models

\title{
5. Conclusion
}

So far very petty literature is available on the application of soft computing technique and the comparison of ANN networks. In this paper an attempt has been made to do the comparative study of the networks \& to develop the predicting models by soft computing technique and evaluating the best network in terms of performance of each network. Thus, Artificial Intelligence neural network models hinged on feed forward and cascade forward algorithms were developed for prediction of KW, OC, DD and MRR. The performances of both the models were compared with each other in terms of their predictive accuracy. Cascade forward back propagation artificial intelligence models exhibited best results compared to feed forward artificial intelligence model as shown in Fig 6, 7, $8 \& 9$ and Table 5. But it should be noted that the proposed scheme can be employed merely to compare a set of neural networks nevertheless it does not offer a neural network model selection. We may encounter performance differences with relatively high power as a result of non-linearity in neural network models. We have compared the predictive accuracies among both the models and CF model outperformed overall predictive accuracies.

\author{
Nomenclature \\ FB Feed forward back propagation \\ CF Cascade forward back propagation \\ Ton Pulse on Time \\ Toff Pulse off Time \\ WT Wire Tension \\ WF Wire Feed \\ SV Servo Voltage \\ KW Kerf Width \\ OC Over Cut \\ DD Dimensional Deviation
}


MRR Material Removal Rate

D Wire diameter

\section{References}

Badde D.S., Gupta A.K., Patki V.K., 2012. Cascade and feed forward back propagation artificial neural network models for prediction of compressive strength of ready mix concrete, Second International Conference on Emerging Trends in Engineering (SICETE), Dr. J.J. Magdum College of Engineering, Jaysingpur, India, Vol. 3, pp. 1-6.

Bobbili R., Madhu V., Gogia A.K. 2015, An experimental investigation of wire electrical discharge machining of hot-pressed boron carbide, Defence Technology, Vol. 11, No. 4, pp. 344-349.

Di S., Chu X., Wei D., Wang Z., Chi G., Liu Y. 2009, Analysis of kerf width in micro-WEDM, International Journal of Machine Tools and Manufacture, Vol. 49, No. 10, pp. 788-792.

Goyal S. and Goyal G.K., 2011, Cascade and feedforward backpropagation artificial neural network models for prediction of sensory quality of instant coffee flavoured, Canadian Journal on Artificial Intelligence, Machine Learning and Pattern Recognition Vol. 2, No. 6, pp. 78-82.

Maher I., Ling L.H., Sarhan A.A.D., Hamdi M., 2015, Improve wire EDM performance at different machining parameters ANFIS modelling, IFAC-PapersOnLine, Vol. 48, No. 1, pp. 105-110.

Mathew B., Benkim, Babu J., 2014, Multiple process parameter optimization of WEDM on AISI304 using Taguchi grey relational analysis, Procedia Materials Science, Vol. 5, pp. 1613-1622.

Mehrotra K., Mohan C. and Ranka S., 1997. Elements of Artificial Neural Network, Cambridge MIT Press

Ramakrishnana R, Karunamoorthy L. 2008. Modeling and multi-response optimization of Inconel 718 on machining of CNC WEDM process, Journal of Materials Processing Technology, Vol. 207, pp. 343-349.

Rao T.B., Krishna A.G., 2013, Simultaneous optimization of multiple performance characteristics in WEDM for machining ZC63/SiCp MMC, Advanced Manufacturing, Vol. 1, pp. 265-275.

Roberts E., 2017, Neural networks. Course notes, Accessed 16 $6^{\text {th }}$ May 2017, Retrieved from http://cs.stanford.edu/people/eroberts/courses/soco/projects/neural-networks/Architecture/feedforward.html

Vasudevan D., Selladurai V., Nagaraj P., 2004, Determination of closed form solution for acceptance sampling using ANN, Quality Assurance, Vol. 11, No. 1, pp. 43-61.

\section{Biographical notes}

Vaishali Sharma and Manish Kumar Sagar are of the Department of Mechanical Engineering, Madhav Institute of Technology \& Science, Gwalior, India.

Received August 2016

Accepted November 2016

Final acceptance in revised form February 2017 